\title{
An Improved LEACH Routing Algorithm Based on WSN
}

\author{
Wang Bo \\ Chongqing College of Electronic Engineering, Chongqing, 401331, China \\ 1277796604@qq.com
}

Keywords: Wireless sensor network; LEACH protocol; E-LEACH protocol; NS simulation.

\begin{abstract}
The number of wireless sensor network nodes is huge and the energy of a single node is limited, so the primary goal of routing protocol design is to improve energy efficiency and prolong the network lifetime. This paper chooses LEACH routing protocol as the research focus, analyzes the implementation of the routing algorithm, and proposes an improved E-LEACH routing algorithm based on the characteristics of sensor node energy and network lifetime. The NS2 simulation software is used to simulate the routing protocol, and the validity of the protocol is verified.
\end{abstract}

\section{Introduction}

Wireless Sensor Network (WSN) is a hot research topic in the world at present, it combines the cross-application of computing, communication and sensor, and has a very broad application prospect. In network, data transmission is controlled by routing protocol. Therefore, it is inseparable from the research of routing protocols to design an excellent network system. Wireless sensor networks have the following characteristics: (1) Nodes do not have a unique global identifier, traditional Internet routing protocols can not be used in sensor networks. (2) All nodes in the sensor network are source nodes, sending data to the only destination node, Sink. (3) Because a large number of nodes are deployed inside or near the object under test, the data collected by them are the same or similar. This requires routing protocols to have data fusion capability. (4) Nodes have limited processing power, power and storage capacity. Developing routing protocols for WSN has become a hot and difficult topic in wireless sensor networks.

\section{Brief introduction of WSN}

\subsection{WSN concept}

Wireless sensor network (WSN) is a network composed of a large number of small sensors. These small sensors are commonly called sensor node. In such networks, one or more base stations (called sinks) are also used to concentrate data collected from small sensors.

\subsection{Structure of WSN}

\subsubsection{Structure of node}

In different applications, the structure of sensor nodes is different, but generally they are composed of sensor module, processor module, wireless communication module and energy supply module.

Sensor module is responsible for the collection and data conversion of the information in the monitoring area. The type of sensor is determined by the form of the physical signal being monitored, such as platinum resistance sensor for temperature monitoring, capacitive sensor for pressure sensing, etc. Processor module is responsible for controlling the operation, storage and processing of the entire sensor node. The wireless communication module is responsible for wireless communication with other sensor nodes, exchanging control information and receiving and receiving data. The energy supply module provides the energy needed for the operation of sensor nodes. 


\subsubsection{Network architecture}

The typical architecture of wireless sensor networks, as shown in Figure 1, includes a large number of randomly distributed sensor nodes, sink nodes, Internet, user terminals, etc.

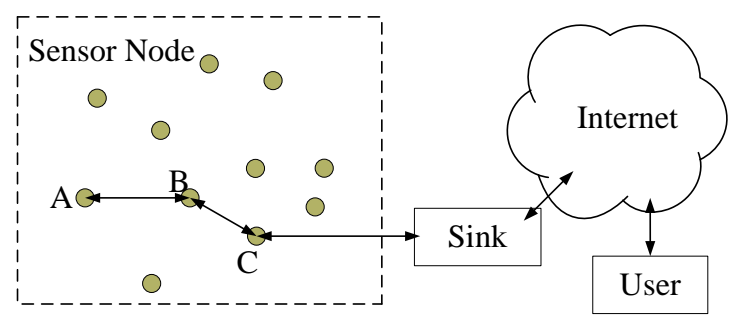

Fig.1 Structure of wireless sensor network

Sensor nodes scattered in the monitoring environment send data to the sink node by network self-organizing multi-hop mode. The sink node can communicate with the external network in many ways, such as Internet satellite or mobile communication network, etc., to transmit the data in the whole area to the user terminal, and the user can manage the node to no one. Line sensor network is configured and managed.

\section{Analysis and research of LEACH routing protocol}

LEACH is the first hierarchical routing protocol proposed in WSN. Many of the following hierarchical routing protocols are based on it. So here we introduce the LEACH protocol.

\subsection{Model of LEACH protocol}

LEACH is a low-power adaptive clustering routing algorithm designed by Handrakasan et al. for wireless sensor networks. Compared with the general plane multi-hop routing protocol and static clustering algorithm, LEACH can extend the network life cycle by $15 \%$, mainly by randomly selecting cluster leaders and sharing the relay traffic equally. LEACH defines the concept of "round", which consists of two stages: initialization and stabilization. In order to avoid additional processing overhead, stability generally lasts for a relatively long time, as shown in Figure 2.

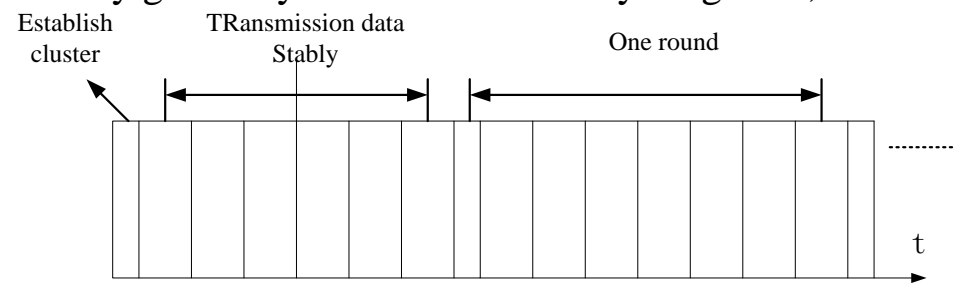

Fig. 2 The sequence diagram of LEACH protocol

In the initialization stage, the cluster leader is generated through the following mechanism. The sensor node generates a random number between 0 and 1 . If the number is greater than the threshold $\mathrm{T}$, the method of selecting the sensor node as the cluster leader $\mathrm{T}$ is as follows:

$$
T=\frac{P}{1-P[r \bmod (1 / p)]}
$$

In Formula (1),P is the percentage of the cluster leader in the node, and $\mathrm{r}$ is the current number of rounds.

When the cluster head is selected, the cluster head node actively broadcasts the message (ADV_CH) in the network that it becomes the cluster head. The nodes that receive this message select the cluster it wants to join according to the strength of the received signal and notifies the corresponding cluster head with a concurrent message (JOIN_REQ). Based on time division multiple access (TDMA), the cluster head node allocates communication slots for each member of the cluster and notifies all intra-cluster nodes through broadcast. This ensures that each node in the 
cluster carries out data transmission in a specified transmission slot, and enters a dormant state at other times, this mechanism can reduce energy consumption. In the stable working stage, the node continuously collects monitoring data and transmits the monitoring data to the cluster head node when the transmission slot arrives. The cluster head node sends the received data to the Sink node after fusion processing, which is a reasonable mode to reduce communication traffic. After a period of time, the whole network enters the next round of work cycle and chooses the cluster head node again.

LEACH protocol uses dynamic cluster head conversion to average the energy consumption of the network nodes, which makes the nodes that fail due to energy exhaustion randomly distributed. Therefore, compared with the general multi-hop routing protocol and static cluster algorithm, LEACH can extend the network lifetime by $15 \%$. However, LEACH protocol spends a lot of money on cluster heads during the process of clustering after each round of fixed cluster heads. It is possible that cluster head nodes are located at the edge of the network or adjacent to several cluster head nodes. Some nodes have to transmit a long distance to communicate with the cluster head, which leads to a large amount of energy consumption. Moreover, all cluster heads of LEACH protocol communicate directly with Sink nodes, adopting continuous data transmission mode and single-hop path selection mode, which makes the cluster heads in each round consume enormous energy, so it is not suitable for large-scale sensor networks.

\subsection{Disadvantages of LEACH protocol}

LEACH algorithm is the first hierarchical network topology organization algorithm proposed for wireless sensor networks. Compared with the way nodes send data directly to BS, the performance of LEACH algorithm is greatly improved. However, the algorithm has some limitations in some aspects, it is mainly reflected in:

The cluster head not only receives and fuses the data collected by the member nodes in the cluster, but also sends the data to BS. Therefore, the energy consumed by the cluster head is more than that of the ordinary member nodes, and it is easy to fail, which leads to frequent clustering. This not only consumes more energy, but also generates a lot of head overhead, this leads to lower utilization of node energy.In addition, the LEACH protocol is only applicable to small scale wireless sensor networks. Because the cluster members send the original data directly to the cluster head through a single hop, and the cluster head sends the fused data to the BS through a single hop, the cluster members and the cluster head, and the BS must be placed within the communication reachable range.

\subsection{LEACH improved E-LEACH protocol}

A heterogeneous-aware protocol called E-LEACH is proposed in this paper, which can prolong the dead time of the first node, in order to prolong the stability period, E-LEACH protocol tries to maintain a balanced energy consumption, usually, the probability that E-LEACH protocol chooses the node with high initial energy to become the cluster head is higher than that of the normal node, which is equivalent to the constraints on energy consumption.

E-LEACH protocol assumes that each node knows the total energy of the network, and then calculates the optimal probability of becoming the cluster head according to the residual energy of the node. At the beginning, each node is assigned a weight $P_{\text {opt }}$ corresponding to the optimal probability, the weight must be equal to the initial energy of each node divided by the initial energy of the ordinary node, it is also defined $P_{n r m}$ as the probability of weighted election of ordinary nodes, and $P_{a d v}$ as the probability of weighted election for advanced nodes.

$$
\begin{aligned}
P_{n r m} & =\frac{P_{o p t}}{1+a} \\
P_{a d v} & =\frac{P_{o p t}}{1-a}(1+a)
\end{aligned}
$$


In Formula (3), a is the initial energy of the advanced node, its value is the multiple of the initial energy of the ordinary node, $\mathrm{m}$ is the proportion of the advanced node in the total number of nodes. The thresholds of ordinary nodes and advanced nodes as cluster heads are $T\left(S_{n r m}\right)$ and $T\left(S_{a d v}\right)$ respectively, and the formula is as follows:

$$
\begin{aligned}
T\left(S_{n r m}\right) & \left.\left.=\frac{P_{n r m}}{1-P_{n r m}[r \bmod (1 / p r r m}\right)\right] \\
T(\operatorname{Sadv}) & =\frac{P_{a d v}}{1-P_{a d v}[r \bmod (1 / p a d v)]}
\end{aligned}
$$

In Formula (4), $\mathrm{r}$ is the current round, this ensures that nodes with high residual energy become cluster heads with larger probability than those with low residual energy. Compared with the traditional LEACH, E-LEACH protocol has the following advantages:

(1) Different nodes have different initial energy, E-LEACH protocol chooses the cluster head with large energy to prolong the death time of the first node, that is, to prolong the stability period.

(2) Due to the extension of the stability period, the throughput of the E-LEACH protocol is also higher than that of other clustering protocols.

(3) In an ideal phase, the energy of E-LEACH protocol is evenly distributed on all nodes in the sensor network.

\section{LEACH and E-LEACH protocol NS2 simulation comparison}

To compare the performance of LEACH and E-LEACH, we generate a $100 \mathrm{~m} * 100 \mathrm{~m}$ region in NS2 simulation environment, and randomly generate a connected graph with 100 nodes. Assuming that each node can perceive the type of traffic that needs to be transmitted before the network is formed, Sink nodes in the network area, and do not consider the energy consumption of Sink nodes, the initial energy of ordinary nodes is $80 \mathrm{~mJ}$, and 15 nodes are advanced nodes. the experimental results are shown in Fig.3.

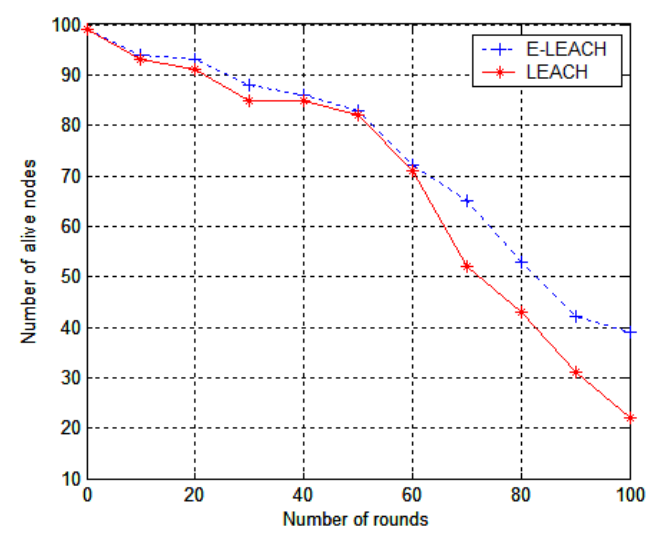

Fig.3 The amount of alive node of LEACH protocols and E-LEACH protocols

As can be seen from Figure 3, the first node of LEACH protocol dies faster than the E-LEACH, that is, the stability period of E-LEACH protocol is longer. This is because the E-LEACH protocol uses the weighted cluster head selection method, the probability of high-level nodes with high initial energy becoming cluster heads is higher, and the time of death will be longer.

\section{Conclusions}

This paper analyzes the current research on Routing in sensor networks, classifies the common routing algorithms, describes the existing algorithms in detail, and describes their respective advantages and disadvantages. The classical hierarchical routing algorithm LEACH is analyzed and studied. Aiming at the characteristics of sensor's first node's death time, an improved E-LEACH 
algorithm is proposed. The E-LEACH algorithm successfully prolongs the probability that the sensor will become a cluster head with high residual energy by setting different initial energy for the node and then selecting cluster head with weights. The death time of the first node is to extend the stability period and increase the throughput.

\section{References}

[1] CHEN Chen, YANG Hong-Li. Energy Consumption Improvement of LEACH in WSNs [J].Computer Systems \& Applications, 2017, 26(11):205-212.

[2] WU Biao, YU Jian, YI Ren-jie. A time-division and Clustering algorithm of LEACH Based on Residual Energy of Sensor Nodes[J].Fire Control \& Command Control,2016,41(10):84-88

[3] WANG Lin, ZHAO Shao-ying. Research and improvement about LEACH routing protocol for wireless sensor networks [J].Computer Engineering and Applications, 2012, 48(2):80-82.

[4] CHEN Shu, XU Yuan. Improved LEACH Protocol Based on Clustering and Coverage Optimization [J].Computer Engineering, 2014, 40(11):97-100. 